\title{
Demethylation of miR-9-3 and miR- 193a Genes Suppresses Proliferation and Promotes Apoptosis in Non-Small Cell Lung Cancer Cell Lines
}

\author{
Jinliang Wang Bo Yang Lu Han Xiaoyan Li Haitao Tao Sujie Zhang Yi Hu \\ Department of General Oncology, Chinese PLA General Hospital, Beijing, China
}

\section{Key Words}

miR-9-3 • miR-193a $•$ Methylation $\cdot$ Demethylation $•$ Non-small-cell lung carcinomas (NSCLCs)

- Cancer • Proliferation • Apoptosis • Gene expression • Signaling pathway

\begin{abstract}
Background: MicroRNAs miR-9-3 and miR-193a have recently been found to be hypermethylated in a variety of non-small cell lung cancer (NSCLC) cells and primary human tumors. The objectives of this study were to investigate the role of demethylation of miR-9-3 and miR-193a genes in regulating proliferation and apoptosis in NSCLCs, and to decipher the potential mechanisms underlying the properties. Methods: MTT and population doubling time by flow cytometry were used to assess cell proliferation. Enzyme-Linked Immunosorbent Assay and caspase-3 activity assay were employed to evaluate apoptosis. Real-time RTPCR and Western blot were used to quantify gene expression at mRNA and protein levels, respectively. Methylation-specific PCR was utilized to assess methylation status. Results: We found that demethylation agent 5 -Aza-2'-deoxycytidine (5-AzaC) reduced cell numbers and prolonged population doubling time (PDT), and promoted doxorubicin-induced apoptosis in seven NSCLC cell lines with different methylation statuses on miR-9-3 and miR-193a promoter regions: NCI-H1993/NCI-H1915 (miR-9-3+/miR-193a+), NCI-H1975/NCI-H200 (miR-9-3+ / miR-193a-), A427/NCI-H2073 (miR-9-3-/miR-193a+), and NCI-H1703 (miR-9-3-/miR-193a-). Treatment with 5-AzaC concomitantly upregulated expression of miR-9-3 and miR-193a, and downregulated their respective target genes NF-KB and Mcl-1. The effects of 5-AzaC were abolished by concomitant knockdown of miR-9-3 and miR-193a using the complex antisense technique, whereas forced ectopic expression of miR-9-3 and miR-193a mimicked the effects of 5-AzaC. We further observed that the strength of proliferation inhibition and apoptosis promotion elicited by 5-AzaC was in the order of NCI-H1993/NCI-H1915 > A427/NCI-H2073 > NCI-H1975/NCI-H200 > NCI-H1703. Conclusions: Methylation-silencing of miR-9-3 and miR-


193a may be an important epigenetic mechanisms favoring NSCLC cell growth and survival for carcinogenesis and cancer progression, and demethylation to reactivate expression of miR-93 and miR-193a genes contributes, at least partially, to the anti-cancer properties of 5-AzaC and thereby may be worthy of future studies for the possibility of being a new therapeutic strategy for the treatment of human NSCLCS.

Copyright $\odot 2013$ S. Karger AG, Basel

\section{Introduction}

Non-small-cell lung carcinomas (NSCLC), a class of epithelial lung cancer including squamous cell carcinoma, large cell carcinoma, and adenocarcinoma, is the leading cause of cancer-related death in Western countries. Despite the development of novel molecular therapies, the majority of patients with advanced or metastatic disease show rapid progression and a median survival time of $<18$ months. NSCLC is relatively insensitive to chemotherapy, compared to small cell carcinoma. The last decade witnessed significant progress in improving our understanding of the biology of NSCLC, which led to the identification of several genetic and epigenetic targets including microRNAs (miRNA), a class of non-protein-coding RNAs, which play a central role in regulating expression of genes related to NSCLC.

Cancer is a complex disease with extensive genetic and epigenetic defects. The role of DNA methylation, a major form of epigenetic modifications, in the development of tumorigenesis has long been recognized. DNA methylation is known to be a part of the epigenetic gene regulatory machinery, resulting from the covalent addition of a methyl group to the $5^{\prime}$ carbon of cytosines within cytosine-guanine (CG) dinucleotides [1], which are enriched in certain genomic regions, called CpG islands (CGI) [2]. CpG islands are found in approximately $60 \%$ of the human gene promoter regions, including both protein coding and non-protein-coding genes such as miRNA genes [3, 4]. Recent studies have highlighted the potential role of epigenetic silencing of miRNA genes in the development of cancers and as a useful biomarker for cancer detection. In lung cancer, numerous tumor suppressor genes are methylated and thus, transcriptionally silenced [4-8]. Strikingly, two recent genomewide studies revealed that an impressive number of miRNAs is methylated in NSCLC cell lines and primary tumors as well $[9,10]$. Among these, miR-9-3 and miR-193a stand out to be the two most frequently identified miRNAs with methylation in their promoter regions. Similar results have also been reported by other studies $[11,12]$. For example, Kitano and colleagues [12] observed miR-9-3 methylation in 65\% of primary NSCLCs. Consistent with these observations, expression downregulation of the precursors of miR-9-3 and miR-193a in NSCLCs have been documented by several laboratories [9-15]. Most notably, miR-93 and miR-193a were found to be tumor specifically methylated in patients with NSCLC, and a shorter disease-free survival of patients was associated with miR-9-3 methylated lung squamous cell carcinoma (LSCC) than patients with miR-9-3 unmethylated LSCC and a shorter overall survival of patients with miR-9-3 methylated LSCC than patients with miR-9-3 unmethylated LSCC [9]. These findings suggest that methylation is an important mechanism for inactivation of certain miRNAs, particularly miR-9-3 and miR-193a in NSCLCs and that miR-9-3 methylation may serve as a prognostic parameter in patients with LSCC.

However, how miR-9-3 and miR-193a methylation affects NSCLC remained unknown. It has been reported that miR-9-3 and miR-193a repress expression of certain oncogenic factors, such as E-cadherin, c-kit, CDK6, and E2F6, which are predicted to enhance tumor angiogenesis and invasiveness [16-19]. Based on computational prediction by miRNA databases, miR-9-3 and miR-193a have the potential to target the anti-apoptotic NF- $\kappa$ B and the anti-apoptotic myeloid leukemia cell sequence-1 (MCL1), respectively, which are known to be the crucial factors in determining cell proliferation and apoptosis [20-25].

DNA methylation is reversible by demethylation, the process of removal of a methyl group from nucleotides in DNA. The DNA methyltransferase inhibitor 5-aza-2'-deoxycytidine (5-AzaC) is the most commonly used demethylating drug able to reverse DNA methylation 
resulting in gene reexpression. Moreover, a synergistic effect of 5-AzaC and the histone deacetylase inhibitor trichostatin A (TSA) in gene reexpression of epigenetically silenced genes has been documented [26]. Use of these agents robustly increased expression of miR-9-3 and miR-193a in NSCLC, presumably by demethylation. These demethylating agents provide a unique and efficient pharmacological probe for studying the role of miRNA demethylation in NSCLC.

These findings prompted us to propose that demethylation of miR-9-3 and miR193a genes may produce proliferation-inhibiting and apoptosis-promoting effects due to repression of NF-KB and MCL1 by miR-9-3 and miR-193a. This study was conducted to test our hypothesis in seven NSCLC cell lines with varying methylation statuses of both miR-9-3 and miR-193a.

\section{Materials and Methods}

\section{Cell culture}

The NSCLC cell lines A427, NCI-H1975, and NCI-H1993 were purchased from the American Type Culture Collection (Manassas, VA) and were grown in $5 \% \mathrm{CO}_{2}$ at $37^{\circ} \mathrm{C}$ in RPMI 1640 medium with high glucose (4.5 g/L; GIBCO-BRL, MD), supplemented with $10 \%$ fetal bovine serum (Hyclone, Logan, Utah). The cell lines were chosen according to their methylation status of miR-9-3 and miR-193a: NCI-H1993 and NCI-H1915 (miR-9-3+/miR-193a ${ }^{+}$), NCI-H1975 and NCI-H200 (miR-9-3/miR-193a-), NCI-H2073 and A427 (miR-9-3-/miR-193a+), and NCI-H1703 (miR-9-3-/miR-193a-), based on the study reported by Heller et al. [9].

\section{Drug treatment}

To achieve DNA demythylation, a total of $2 \times 10^{5} \mathrm{NSCLC}$ cells $/ \mathrm{mL}$ were treated with $0.5 \mu \mathrm{mol} / \mathrm{L} 5$-Aza2 '-deoxycytidine (5-AzaC, Sigma, St. Louis, MO) for 3 days, during which medium was replaced daily. Control cells were not drug treated.

To induce apoptotic cell death, NSCLC cells in culture were incubated with doxorubicin $(0.5 \mu \mathrm{mol} / \mathrm{L})$ for $48 \mathrm{~h}$ [27].

\section{MTT assay for cell proliferation}

The WST-1 kit (Roche, Penzberg, Germany) was used to assess cell survival according to the manufacturer's instructions. In brief, $24 \mathrm{~h}$ after treatment with varying drugs or constructs, NSCLC cells were washed with PBS and then grown in $100 \mu \mathrm{l}$ of fresh culture medium addition of $10 \mu \mathrm{l}$ of WST-1 reagent for $30 \mathrm{~min}$. The absorbance was measured at $425 \mathrm{~nm}$ using a Spectra Rainbow microplate reader (Tecan, Grödig, Austria) with a reference wavelength of $690 \mathrm{~nm}$.

Determination of population doubling time (PDT)

Cell proliferation was determined by characterizing the log phase growth with population doubling time (PDT) which was calculated by using the equation: $1 /\left(3.32 \times\left(\log N_{\mathrm{H}}-\log N_{\mathrm{I}}\right) /\left(t_{2}-t_{1}\right)\right.$, where $N_{\mathrm{H}}$ is the number of cells harvested at the end of the growth period $\left(t_{2}, 72 \mathrm{~h}\right)$ and $N_{\mathrm{I}}$ is the number of cells at $5 \mathrm{~h}\left(t_{1}\right)$ after seeding [28]. Cells were counted by a flow cytometer (EPICS XL; Beckman, Inc.).

\section{Enzyme-Linked Immunosorbent Assay (ELISA)}

The Cell Death Detection ELISA kit (Roche Molecular Biochemicals) was employed to quantify DNA fragmentation of apoptotic cells on the basis of antibody detection of free histone and fragmented DNA, as detailed elsewhere $[27,29]$. Optical density value was read by a microplate reader (GloMax@-96 Microplate Luminometer, Promega).

\section{Caspase- 3 activity assay}

We used the Caspase-3 Colorimetric Activity Assay Kit (Merck Millipore) to measure caspase-3 activities by following the manufacturer's instructions. Samples after complete reactions were read at $405 \mathrm{~nm}$ in a microtiter plate reader (GloMax®-96 Microplate Luminometer, Promega). Fold-increase in 
caspase-3 activity was determined by comparing the OD reading from the induced apoptotic sample with the level of the un-induced control.

\section{Quantitative real-time RT-PCR analysis}

The total RNA samples were isolated with Ambion's mirVana miRNA Isolation Kit, from NSCLC cells. The mirVana ${ }^{\mathrm{TM}}$ qRT-PCR miRNA Detection Kit (Ambion) was employed with real-time PCR with TaqMan for quantification of miRNAs. RT Reactions contained mirVana qRT-PCR primer sets specific for human miR-9-3 and miR-193a, and a scrambled miRNA as a negative control. qRT-PCR was performed on a thermocycler ABI Prism ${ }^{\circledR} 7500$ fast (Applied Biosystems) for 40 cycles. Fold variations in expression of an mRNA between RNA samples were calculated. The threshold cycle $\left(\mathrm{C}_{\mathrm{T}}\right)$ is defined as the fractional cycle number at which the fluorescence passes the fixed threshold.

\section{Western blot analysis}

The protein samples (cytosolic) were extracted from cultured NSCLC cells for immunoblotting analysis. Protein samples were quantified by BCA Protein Assay Kit with bovine serum albumin as the standard. Each protein sample at an amount of $50 \mu \mathrm{g}$ was fractionated by SDS-PAGE (12\% polyacrylamide gels) and then transferred to PVDF membrane (Millipore, Bedford, MA). The sample was then incubated overnight at $4^{\circ} \mathrm{C}$ with the primary antibodies in 1:1200: affinity purified goat polyclonal anti-NF- $\mathrm{kB}$ (p65) (Santa Cruz Biotechnology Inc.) and goat polyclonal anti-MCL1 (Santa Cruz Biotechnology Inc.). Next day, the membrane was incubated with secondary antibodies (Molecular Probes) diluted in PBS for $2 \mathrm{~h}$ at room temperature. Finally, the membrane was rinsed with PBS before scanning using the Infrared Imaging System (LI-COR Biosciences). GAPDH was used as an internal control for equal input of protein samples, using anti-GAPDH antibody (Santa Cruz Biotechnology Inc.). Immunoblot band density was quantified using QuantityOne software by measuring the band intensity (Area $\times$ OD) and normalizing to GAPDH. Relevant data are expressed as fold changes by normalizing the data to the control values.

\section{Synthesis of miRNAs and anti-miRNA antisense inhibitors}

miR-9-3 (5'-UCU UUG GUU AUC UAG CUG UAU GA-3'), miR-193a (5'- AAC UGG CCU ACA AAG UCC CAG U-3'), their respective antisense oligonucleotides AM0-9-3 (5'-TCA TAC AGC TAG ATA ACC AAA GA-3') and AMO-193a (5'-ACT GGG ACT TTG TAG GCC AGT T-3'), the complex antisense inhibitors targeting both miR9-3 and miR-193a (cAMO: 5'-ACT GGG ACT TTG TAG GCC AGT T tttttTCATACAGCTAGATAACCAAAGA-3'),

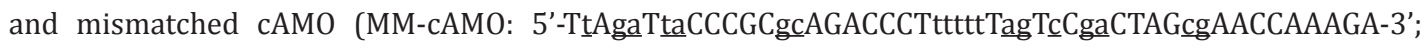
the underlined low-case letters indicate nucleotide replacement) [30] were synthesized by Integrated DNA Technologies Inc (IDT). Five nucleotides or deoxynucleotides at both ends of the antisense molecules were modified with the ribose ring being constrained by a methylene bridge between the 2'-O-and the 4'-C atoms to enhance cellular stability and target affinity [31].

\section{Transfection procedures}

NSCLCs were transfected with miRNA (10 nmol/L), cAMO (2 nmol/L), AMO-9-3 $(2 \mathrm{nmol} / \mathrm{L})$ or AMO-193a ( $2 \mathrm{nmol} / \mathrm{L})$, or negative control AMOs, with lipofectamine 2000 (Invitrogen), according to manufacturer's instructions. Forty-eight hours after transfection, cells were used for measuring proliferation and apoptosis, or were collected for total miRNA or protein purification.

\section{DNA methylation analysis}

DNA methylation for miR-9-3 and miR-193a was analyzed by bisulfate conversion of DNA (500 ng/

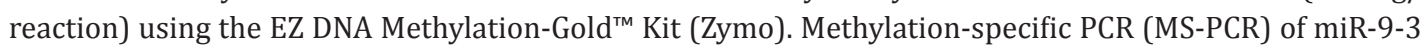
and miR-193a was performed using the GoTaq ${ }^{\mathrm{TM}}$.

DNA Polymerase (Promega) with 30 cycles reactions [32,33]. The primer pairs used for MS-PCR were based on the study reported by Heller et al [9]. For miR-9-3, forward: 5'-GTG YGT GTG TTT GTT TAT TTT-3' and reverse: 5'-ACC TCC CTT AAC CAA TAC C-3'; for miR-193a: forward: 5'-TTT GAG GGA TAT TTA GAG TTT YGG-3' and reverse: 5'-CAA CTC CCA TCC TCR AAA TT-3'. The expected PCR products are $141 \mathrm{~kb}$ for miR-9-3 and $115 \mathrm{~kb}$ for miR-193a. Each reaction was composed of known positive and negative water controls. PCR products were run on a $2.0 \%$ agarose gel and were visualized with UV transillumination (Kodak). 
Fig. 1. Verification of methylation status of seven non-small cell lung cancer (NSCLC) cell lines using methylation-specific PCR (MS-PCR). Shown are agarose gel examples of MS-PCRs of miR-9-1 and miR-193a, respectively, with and without the demethylation agent 5-Aza-2'deoxycytidine (5-AzaC). The appearance of a band indicates the presence of methylation.

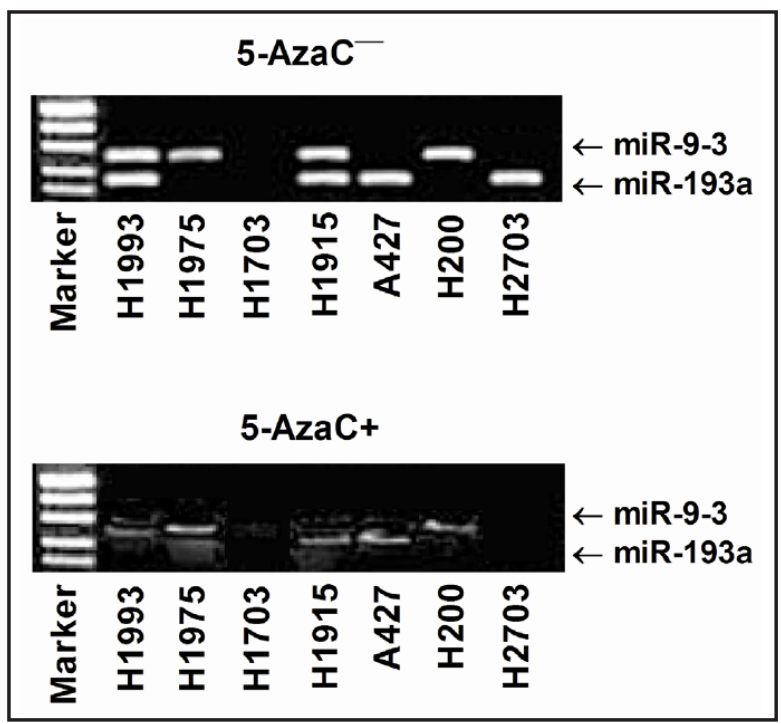

Data analysis

All data are expressed as mean \pm SEM. Statistical comparisons among multiple groups were performed by analysis of variance (ANOVA). If significant effects were indicated by ANOVA, a $t$-test with Bonferroni correction was used to evaluate the significance of differences between individual means. Otherwise, baseline and drug data were compared by paired Student's $t$-test and age-matched comparisons between control and treatment were done by unpaired Student's $t$-test. A two-tailed $p<0.05$ was taken to indicate a statistically significant difference. All statistical tests were performed using SPSS 19.0.

\section{Results}

Verification of methylation status of seven NSCLC cell lines before and after 5-AzaC

Seven NSCLC cell lines were chosen for our study based on their differential miR-93 and mkiR-193a methylation statuses as reported by Heller et al [9]. We first conducted methylation-specific PCR (MS-PCR) analysis to verify the methylation statuses of the seven cell lines under our experimental conditions. As depicted in Figure 1, the appearance of the PCR bands confirmed the statuses as NCI-H1993 and NCI-H1915 (miR-9-3+/miR-193a ${ }^{+}$,

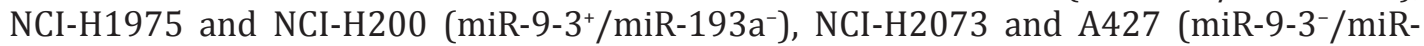
$193 \mathrm{a}^{+}$), and NCI-H1703 (miR-9-3-/miR-193a $\mathrm{a}^{-}$). Moreover, treatment with the demethylation agent 5-AzaC essentially wiped out or substantially weakened the bands.

Inhibition of proliferation and promotion of apoptosis by demethylation agent 5-AzaC

All NSCLC cell lines tested, except for H1703 (miR-9-3-/miR-193a ${ }^{-}$), exposed to 5 -AzaC $(0.5 \mu \mathrm{mol} / \mathrm{L})$ demonstrated significantly reduced cell number and lengthened PDT, as determined by MTT assay and flow cytometry method, respectively, compared with mocked-treated cells (Fig. 2A \& 2B). Intriguingly, the degrees of decrease in cell number and prolongation of PDT were both in the order of NCI-H1993/NCI-H1915 > A427/NCI-H2073 $>$ NCI-H1975/NCI-H200 > H1703.

After treatment with doxorubicin (Dox; $0.5 \mu \mathrm{mol} / \mathrm{L}$ ), apoptotic cell death of NSCLCs was observed, as reflected by the increased optical density (OD) values as an indicator of DNA defragmentation measured by ELISA (Fig. 3C). The percentage of apoptosis was in the order of H1703 > NCI-H1975/NCI-H200 > A427/NCI-H2073 > NCI-H1993/NCI-H1915. The Dox-induced apoptosis in all three NSCLCs was considerably exacerbated in the presence of 5-AzaC. Strikingly, the order of apoptotic cell death according to the severity was converted to NCI-H1993/NCI-H1915 > A427/NCI-H2073 > NCI-H1975/NCI-H200 > H1703 (Fig. 2C). 

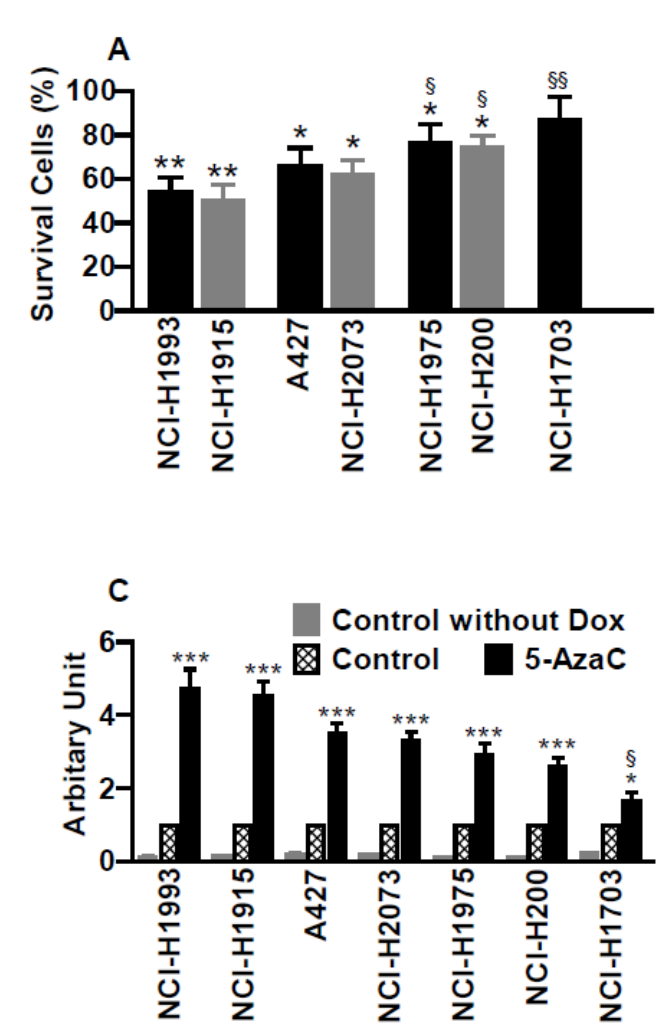
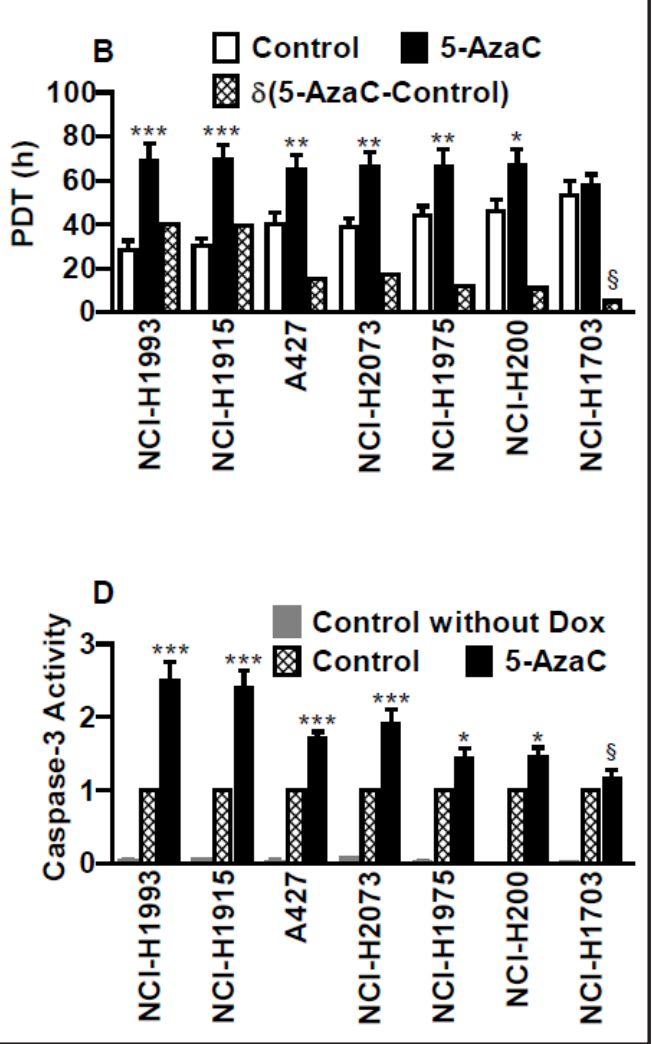

Fig. 2. Inhibition of proliferation and promotion of apoptosis by the demethylation agent 5-Aza-2'deoxycytidine (5-AzaC) in seven NSCLC cell lines. Cells were incubated with 5-AzaC at a final concentration of $0.5 \mu \mathrm{mol} / \mathrm{L}$ for 3 days before measurements. (A) Percent changes of the number of survival cell after 5-AzaC treatment, determined by MTT assay. Cells were counted by flow cytometry methods. (B) Cell proliferation determined by population doubling time (PDT) calculated by using the equation: $1 /\left(3.32 \times\left(\log N_{\mathrm{H}}\right.\right.$ $\left.-\log N_{\mathrm{I}}\right) /\left(t_{2}-t_{1}\right)$, where $N_{\mathrm{H}}$ is the number of cells harvested at the end of the growth period $\left(t_{2}, 72 \mathrm{~h}\right)$ and $N_{\mathrm{I}}$ is the number of cells at $5 \mathrm{~h}\left(t_{1}\right)$ after seeding. $\delta(5$-AzaC-Control) is the difference in PDT values between 5-AzaC and control cells, indicating the length of prolongation of PDT by 5-AzaC. Note that the magnitude of decreases in cell number and prolongation of PDT were in the order of NCI-H1993/H1915 > A427/H2073 > NCI-H1975/H200 > H1703. (C) Apoptotic cell death determined by ELISA quantification of DNA fragmentation. Shown are control-normalized absorbance values. Apoptosis was induced by incubating cells with doxorubicin (Dox; $0.5 \mu \mathrm{mol} / \mathrm{L}$ ) for $48 \mathrm{~h} .{ }^{\S} p<0.05$ ANOVA $F$-test for comparison among the seven cells; ${ }^{*} p<0.05$ vs Ctl, ${ }^{* *} p<0.01$ vs Ctl \& ${ }^{* * * * *} p<0.001$ vs Ctl; $t$-test with Bonferroni correction; $\mathrm{n}=5$ batches of cells/group.

Upregulation of miR-9-3 and miR-193a by demethylation agent 5-AzaC

As the first step to exploit the potential role of altered methylation status of miR-9-3 and miR-193a in mediating the proliferation-inhibiting and apoptosis-promoting effects of demethylation agent 5-AzaC, we quantified the levels of miR-9-3 and miR-193a in all three NSCLC cell lines. Consistent with previously published studies [7], we observed that 5-AzaC robustly upregulated the levels of miR-9-3 and miR-193a in NSCLCs. Notably, the four different groups of NSCLC cell lines showed four distinct patterns of upregulation: miR-9-3 and miR193a were concomitantly and equally increased in NCI-H1993/NCI-H1915; the increase in miR-193a level was substantially greater than in miR-9-3 in A427/NCI-H2073; miR-9-3 was elevated to a greater extent than miR-193a in NCI-H1975/NCI-H200; and neither miR-9-3 nor miR-193a was altered in H1703 (Fig. 3). These data are in line with the study reported by Heller et al [9] unraveling the methylation status of NCI-H1993/NCI-H1915 (miR-9-3+/ 
Fig. 3. Upregulation of miR-9-3 and miR-193a by 5 -AzaC in seven NSCLC cell lines. Note that the different NSCLC cell lines had distinct patterns of upregulation of miR-9-2 and miR-193a: both miRNAs were significantly upregulated in NCI-H1993/ H1915; only miR-193a was significantly upregulated in A427/H2073; only miR-193a was significantly upregulated in NCI-H1975/H200, and neither miR-9-3 nor miR-193a was altered in NCI-H1703. ${ }^{* *} p<0.01$ vs $\mathrm{Ctl} ;{ }^{* * *} p<0.001$ vs $\mathrm{Ctl} ; \mathrm{n}=5$ batches of cells/group.
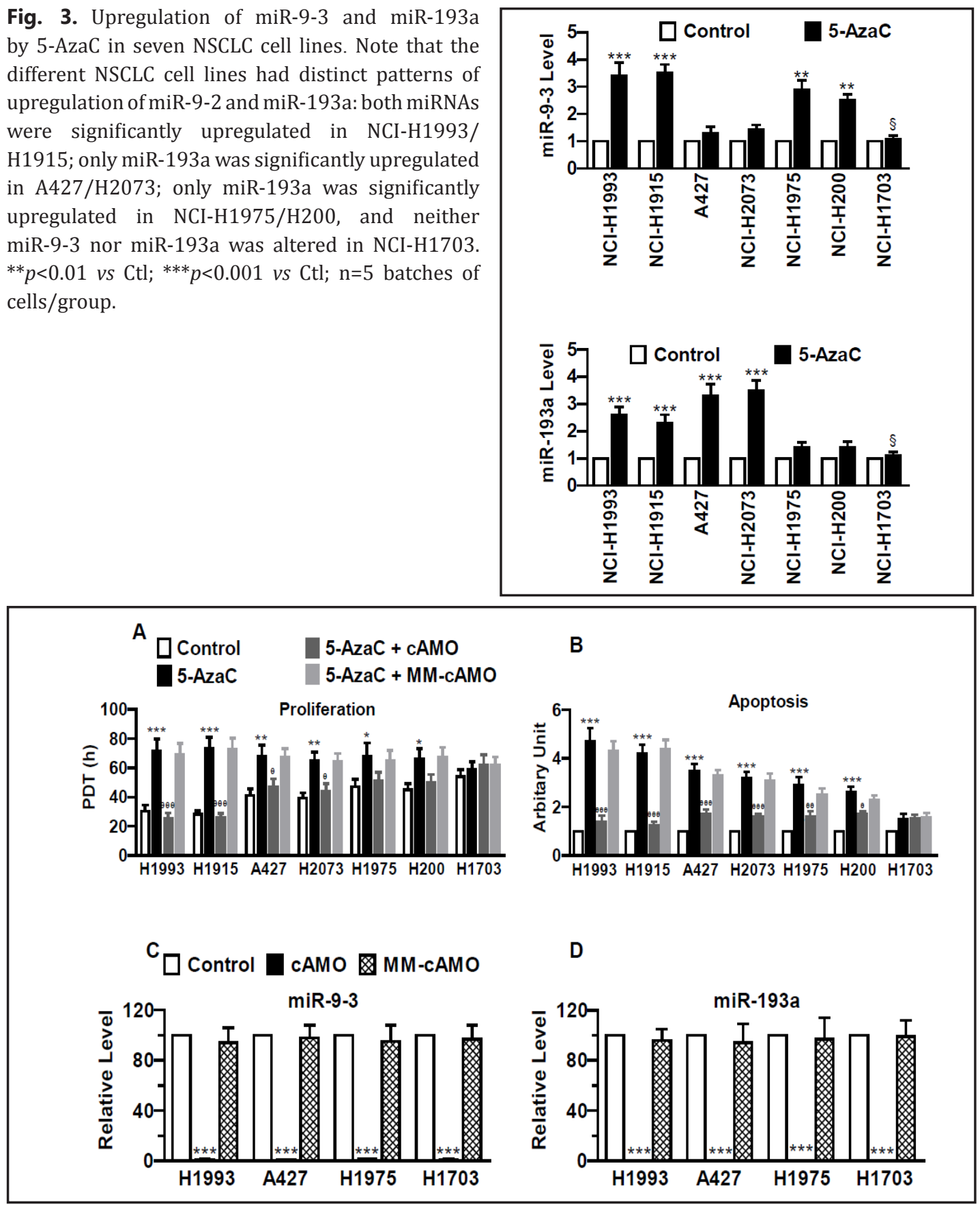

Fig. 4. Cancellation of the proliferation-inhibiting and apoptosis-promoting effects of 5-AzaC by the complex anti-miRNA oligomer (CAMO) in seven NSCLC cell lines. The cAMO was designed to target both miR-9-3 and miR-193a specifically and concomitantly. (A) cAMO reversed the inhibitory effects of 5-AzaC on cell proliferation assessed by PDT. (B) cAMO reversed the promoting effects of 5-AzaC on Dox-induced cell apoptosis assessed by ELISA; Note that the mismatched cAMO (MM-cAMO) as a negative control failed to affect the effects of 5-AzaC on proliferation and apoptosis. (C) Verification of the efficacy of cAMO in knocking down both miR-9-3 and miR-193a, with MM-cAMO for negative control. ${ }^{8} p<0.05$ among the three cell groups by ANOVA $F$-test; ${ }^{*} p<0.05$ vs $\mathrm{Ctl},{ }^{* *} p<0.01$ vs $\mathrm{Ctl} \&{ }^{* * *} p<0.001$ vs $\mathrm{Ctl} ;{ }^{\theta} p<0.05$ vs 5 -AzaC; ${ }^{\theta \theta} p<0.01$ vs 5 -AzaC; ${ }^{\theta \theta \theta} p<0.001$ vs 5 -AzaC by $t$-test with Bonferroni correction; $\mathrm{n}=5$ batches of cells/group.

miR-193a+), A427/NCI-H2073 (miR-9-3-/miR-193a+), NCI-H1975/NCI-H200 (miR-9-3 miR-193a $\mathrm{a}^{-}$, and H1703 (miR-9-3-/miR-193a-). 
Fig. 5. Forced ectopic expression of miR9-3 or miR-193a produces similar effects as 5-AzaC in seven NSCLC cell line. (A) Inhibition of cell proliferation by miR9-3 and miR-193a, as reflected by the prolonged PDT; (B) Enhancement of Doxinduced apoptosis by miR-9-3 and miR$193 \mathrm{a}$, as reflected by the increased DNA fragmentation. ${ }^{*} p<0.05$ vs Ctl, ${ }^{* *} p<0.01$ vs Ctl \& ${ }^{* * *} p<0.001$ vs Ctl; Student $t$-test; $\mathrm{n}=5$ batches of cells/group.

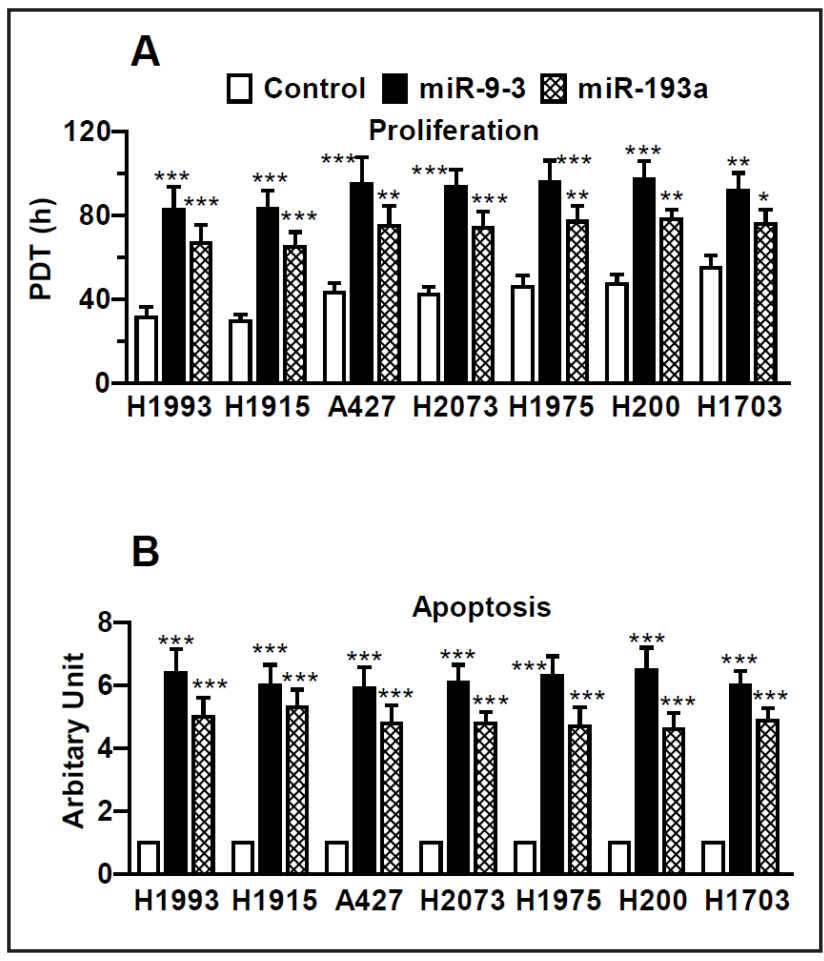

Attenuation of the effects of 5-AzaC by knocking down miR-9-3 and miR-193a

It was anticipated that if upregulation of miR-9-3 and miR-193 is truly involved in proliferation-inhibiting and apoptosis-promoting effects of 5-AzaC, knockdown of these miRNAs in the presence of 5-AzaC should significantly abrogate, if not completely eradicate, the effects of 5-AzaC. Our experiments indeed verified this notion. As illustrated in Fig. 4A, in cells transfected with the complex antisense inhibitors targeting both miR-9-3 and miR193a (cAMO; $2 \mathrm{nM}$ ) substantially mitigated the proliferation-inhibiting effects of 5-AzaC in all three NSCLCs, as indicated by the reversal of PDT lengthening by cAMO in the presence of 5-AzaC. Consistently, the 5-AzaC-evoked enhancement of Dox-induced apoptosis was also pronouncedly attenuated by cAMO (Fig. 4B). It was noted that both the proliferationenhancing and apoptosis-suppressing effects of cAMO were found much greater in NCI-H1993/NCI-H1915 than in other NSCLC cell lines. In all cases, the mismatch cAMO (MMcAMO) failed to alter the proliferation-inhibiting and apoptosis-promoting effects of 5-AzaC.

The efficacy of cAMO in knocking down miR-9-3 and miR-193a was verified, with MMcAMO as a negative control (Fig. 4C, D).

Inhibition of proliferation and promotion of apoptosis by miR-9-3 and miR-193a overexpression

To acquire additional evidence for the role of miR-9-3 and miR-193a upregulation through demethylation by 5 -AzaC in suppressing proliferation and promoting apoptosis of NSCLCs, we examined if forced expression of these two miRNAs is sufficient to elicit the effects in the absence of 5-AzaC. As displayed in Fig. 5, transfection of either of the two miRNAs produced considerable prolongation of PDT and increases in Dox-induced apoptosis in all three cell lines. For a given miRNA, the strength of effects was nearly the same in all three NSCLCs; but between the two miRNAs, the effects of miR-9-3 overexpression appeared to be moderately but significantly greater than miR-193a.

Downregulation of NF- $\kappa B$ and $\mathrm{Mcl}-1$ by demethylation agent 5-AzaC

If demethylation of miR-9-3 and miR-193a genes indeed contributed to the observed effects of 5-AzaC, then the expression their putative target genes should be downregulated. 


\begin{tabular}{|c|c|}
\hline \multirow{5}{*}{$\begin{array}{c}\text { miR-9-3 } \\
\text { AGUAUGUCGAUCUAUUGGUUUCU-5' } \\
\text { | | | | | | | | | | | } \\
\text { UCCCACACCGUGUAAACCAAAGC-52 }\end{array}$} & NFKB Seed Sequence Conservation \\
\hline & Has UCCCACACCGUG----U-AAACCAAAGCCCUAAAAUUCCACU \\
\hline & Ptr UCCCACACCGUG----U-AAACCAAAGCCCUAAAAUUCCACU \\
\hline & Mml UCCCACACCGUA----U-AAACCAAAGCCCUAAAAUUCUACU \\
\hline & Мmu CCCCACACUGUG----U-AAACCAAAGCCCUGACAGUCCAUU \\
\hline \multirow[t]{2}{*}{ NFKB } & Rno CCCCACAC--UG----U-AAACCAAAGCCCUGAAAGGCCAUC \\
\hline & MCL1Seed Sequence Conservation \\
\hline $\operatorname{miR}-193 a$ & Has UUGUUGAGAACAGG-AAAGUGGCCAGUAGCC--AGGCAAGUCAUA \\
\hline UGACCCUGAAACAUCCGGUCAA- 5 , & PtI UUAUUGAGAACAGG-AAAGUGGCCAGUAGCC--AGGCAAGUCAUA \\
\hline 1111111 & Mml UUGUUGAGAACAGG-AAAGUGGCCAGUAGUC--AGGCAAGUCAUA \\
\hline UGAGAACAGGAAAGUGGCCAGUA-338 & Oga UUGUUGAGAUCAGG-AGUGUGGCCAGUAGUU--GGAUUAGUCAUG \\
\hline & The UUGUAUAGAACAGG-AGAGUGGCCAGUAGCC--AGGCUAGUCAUA \\
\hline \multirow{2}{*}{ MCL1 } & Mmu U-AUCUAGAGAUGGAAGAGGGGCCAGUCAUC--AGGCUAGUCACA \\
\hline & Rno UUGUCUGGAGAUGGAAGAGUGGCCAGUCACC--AGGCUAGUCCUA \\
\hline
\end{tabular}

Fig. 6. Sequence alignment between miR-9-3 and NFKB and between miR-193a and MCL1 showing the seed-site complementarity (highlighted in yellow), and sequence alignment of NFKB or MCL1 showing the seed sequence conservation among species (highlighted in yellow).

To test this notion, we went on to analyze the expression of their target genes at the protein level under various conditions. According to computational prediction, NF- $\kappa B$ and Mcl-1 are the candidate target genes for mR-9-3 and miR-193a, respectively (Fig. 6), and NF- $\kappa$ B has been established as a target gene for miR-9 $[34,35]$ and Mcl-1 as a target gene for miR193a [36, 37]. Reciprocal relationships in terms of their expression levels between miR-93 and NF- $\kappa B$, and between miR-193a and Mcl-1 were consistently observed. Specifically, after exposure to 5-AzaC, miR-9-3 and miR-193a were upregulated (Fig. 3) whilst NF-кB and Mcl-1 were downregulated, and these changes were eliminated by cAMO (Fig. 7A). However, distinct patterns of the relationships were observed among the four different

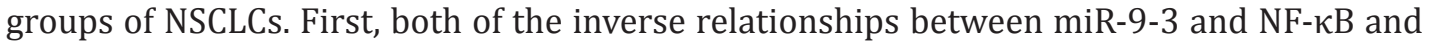
between miR-193a and Mcl-1 were seen in NCI-H1993/NCI-H1915. Secondly, NCI-H1975/ $\mathrm{NCI}-\mathrm{H} 200$ demonstrated only an inverse relationship between miR-9-3 and NF- $\mathrm{KB}$, and A427/NCI-H2073 cells only showed an inverse relationship between miR-193a and Mcl-1. Furthermore, the reciprocal relationship between miR-9-3 and NF- $\kappa B$ was eliminated only by AM0-9-3 (antisense specific for miR-9-3); likewise, the reciprocal relationship between miR-193a and Mcl-1 was eliminated only by AMO-193a (antisense specific for miR-193a) but not by AM0-9-3 (Fig. 7B). Additionally, forced expression of mR-9-3 and miR-193a downregulated NF- $\mathrm{\kappa B}$ and Mcl-1 protein levels, respectively (Fig. 7C).

\section{Discussion}

The objective of this study was to investigate the roles of demethylation of miR-9-3 and miR-193a genes in regulating proliferation and apoptosis in NSCLCs, and to decipher the potential target mechanisms underlying the properties. The main findings of this study include: (1) Demethylation agent 5-AzaC concomitantly upregulated expression of miR-9-3 and miR-193a and downregulated their respective target genes NF- $\mathrm{BB}$ and Mcl-1, accompanied by inhibition of proliferation and promotion of doxorubicin-induced apoptosis in three NSCLCs tested; (2) The effects of 5-AzaC were abolished by concomitant knockdown of miR-9-3 and miR-193a using the complex antisense technique, whereas forced ectopic expression of miR-9-3 and miR-193a mimicked the effects of 5-AzaC; And (3) the degrees of proliferation inhibition and apoptosis promotion elicited by 5-AzaC were both in the order of NCI-H1993 > A427 > NCI-H1975. Collectively, we conclude that methylation of miR-9-3 and miR-193a genes to silence their expression so as to upregulate the anti-apoptotic NF- $\kappa B$ and Mcl-1 may be an epigenetic mechanism for carcinogenesis, and demethylation to re-activate tumor suppressor miRNAs miR-9-3 and miR-193a contributes, at least partially, to the anti- 


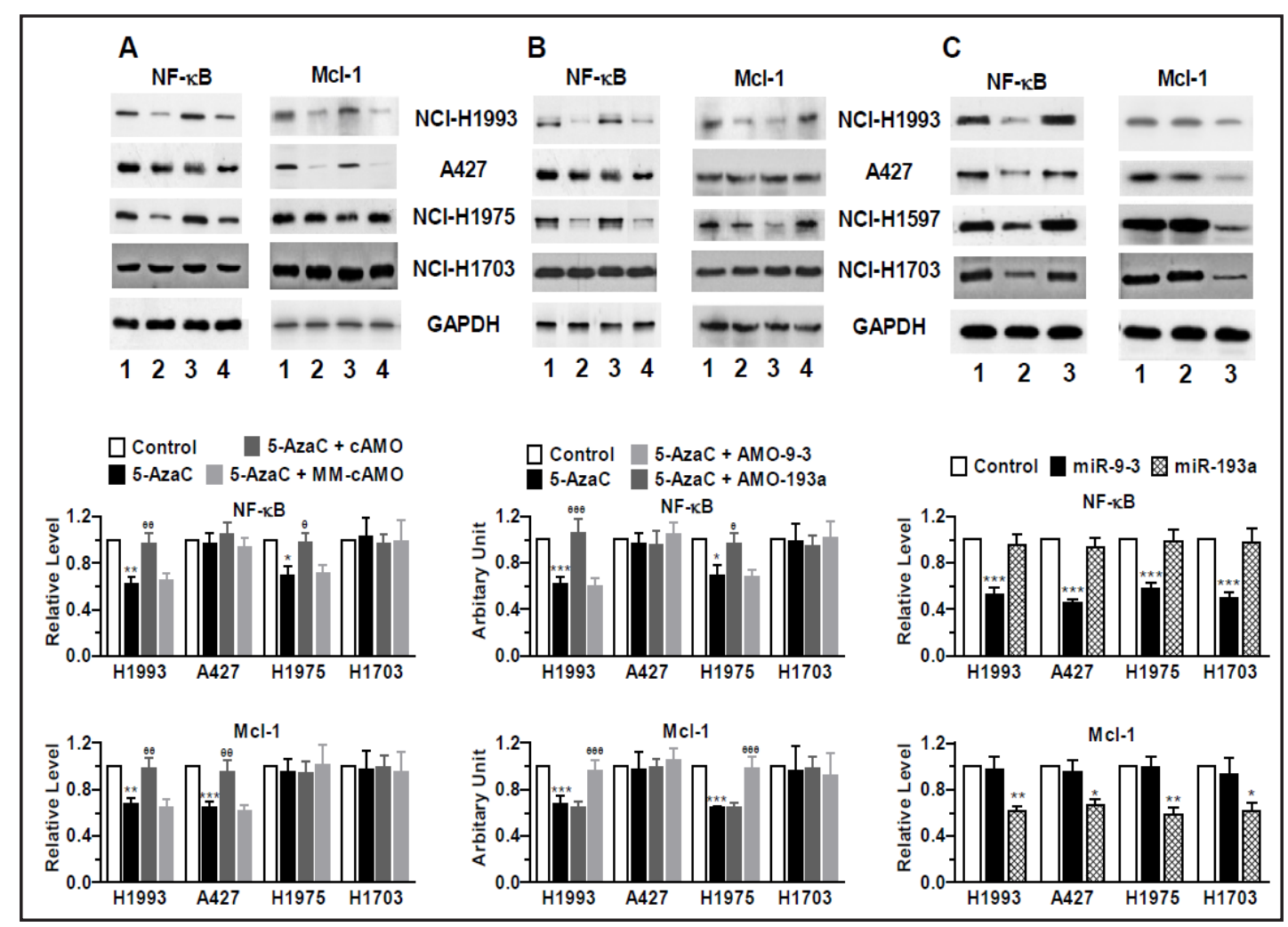

Fig. 7. Altered protein levels of NF- $\mathrm{kB}$ and Mcl-1 under varying conditions in seven NSCLC cell line. Upper panels are examples of Western blot bands and lower panels are mean data of the band density. (A) Effects of 5-AzaC and cAMO on NF- $\mathrm{KB}(65 \mathrm{kDa})$ and Mcl-1 (38 kDa) protein levels. Note that 5-AzaC had no effect on NF- $\mathrm{KB}$ in A427 cells (and H2073, data not shown) and no effect on Mcl-1 in NCI-H1975 (and H200, data not shown). Data from NCI-H1915 (not shown) were similar to those from NCI-H1993. Lane 1: control; lane 2: 5-AzaC; lane 3: 5-AzaC + cAMO; lane 4: 5-AzaC + MM-cAMO; (B) Effects of 5-AzaC and AM0-9-3 or AMO-193a on NF- $\mathrm{KB}$ and Mcl-1 protein levels. Lane 1: control; lane 2: 5-AzaC; lane 3: 5-AzaC + AMO-9-3; lane 4: 5 -AzaC + AMO-193a. ${ }^{\S} p<0.05$ among the seven cell lines by ANOVA F-test; ${ }^{*} p<0.05$ vs Ctl, ${ }^{* *} p<0.01$ vs Ctl \& ${ }^{* * *} p<0.001$ vs Ctl; Student $t$-test; $\mathrm{n}=5$ batches of cells/group; ${ }^{\theta} p<0.05$ vs 5 -AzaC, ${ }^{\theta \theta} p<0.01$ vs 5 -AzaC \& ${ }^{\theta \theta \theta} p<0.001$ vs 5 -AzaC by $t$-test with Bonferroni correction; $n=5$ batches of cells/group. (C) Effects of forced ectopic expression of miR-9-3 or miR-193a on NF- $\kappa$ B and Mcl-1 protein levels. Lane 1: control; lane 2: miR9-3; lane 3: miR-193a. Note that there were no "cross reactivities" between AMO-9-3 or AMO-193a and NF$\kappa \mathrm{B}$ or Mcl-1. ${ }^{\S} p<0.05$ among the seven cell lines by ANOVA $F$-test; ${ }^{*} p<0.05$ vs Ctl, ${ }^{* *} p<0.01$ vs $\mathrm{Ctl} \&{ }^{* * *} p<0.001$ vs Ctl; $\mathrm{n}=5$ batches of cells/group.

cancer properties of 5-AzaC and thereby may be considered a topic for further experimental studies on the possibility as a new strategy for cancer therapy.

One study documented that ectopic expression of miR-9 strongly accelerates terminal myelopoiesis and promotes apoptosis in vitro and in vivo via targeting Fox01 and Fox03 [32]. In another study, ectopic expression of miR-9 was found to inhibit cell proliferation, migration and invasion in gastric cancer cells [33]. Still another study showed that miR-9 regulates E-cadherin and cancer metastasis [30]. We unraveled here that miR-9-3 overexpression of miR-9-3 either by demethylation or by forced expression suppressed proliferation and enhanced apoptosis in NSClCs. In addition to NSCLCs showed in this study and some previous studies [9-15], methylation of miR-9-3 has also been described in a number of cancer cells of other tissue origins, including gastric cancer cells $[33,38]$, hepatocellular carcinoma [39], bladder cancer [40], oropharyngeal squamous cell carcinomas [41], and renal cell carcinoma [42]. Based on our findings in this study, it is not unreasonable to speculate that demethylation of miR-9-3 may also produce anti-cancer effects in other types of cancers. 
Similarly, miR-193a has also been identified as a methylation-silenced tumor suppressor in a variety of cancers [16-18,43]. Moreover, downregulated miR-193a expression was reported in LSCC, malignant melanomas, oral squamous cell carcinomas (OSCC), and acute myelogenous leukemia $[16,18,44,45]$. Together with our findings in this study, it is quite plausible that methylation is also an important mechanism to inactivate miR-193a expression for cancer generation and progression.

We found that doxorubicin evoked only a smaller degree of apoptosis under normal culture conditions, and its proapoptotic property was manifested after cells had been exposed to 5-AzaC for DNA demethylation. Moreover, the apoptosis-promoting effect of 5-AzaC was effectively mitigated by knocking down miR-9-3 and miR-193a. These results suggest that methylation of miR-9-2 and miR-193a contributes to conferring the drug resistance to NSCLCs and demethylation of these miRNAs is able to restore the drug sensitivity of NSCLCs.

The fact that there were consistently distinct patterns in the three different NSCLCs in response to 5-AzaC treatment in terms of proliferation inhibition, apoptosis promotion, upregulation of miR-9-3 and miR-193a, and downregulation of NF- $\kappa \mathrm{B}$ and Mcl-1 protein levels may be best explained by differences in the methylation status of the NSCLCs. NCI-H1993/ NCI-H1915 cells responded with highest sensitivity to 5-AzaC, suggesting that both miR-9-3 and miR-193a are highly methylated in this cell line. By comparisons, NCI-H1975/NCI-H200 and A427/NCI-H2073 appeared to have higher degrees of methylation in miR-9-3 and miR193a, respectively. These results are in agreement with the findings by Heller et al [9] who revealed the methylation status as NCI-H1993/H1915 (miR-9-3+/miR-193a-), NCI-H1975/

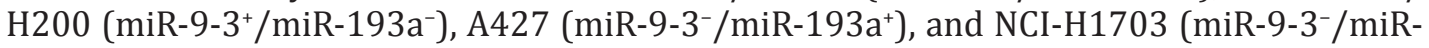
193a-).

Taken together, our study provides strong evidence that methylation-silencing of miR9-3 and miR-193a, as of other miRNAs and protein-coding genes [46-48], is an important epigenetic mechanisms favoring cancer cell growth and survival in NSCLCs and likely in other cancers as well, and demethylation to reactivate expression of miR-9-3 and miR-193a genes may be a novel epigenetic therapeutic strategy for the treatment of human NSCLCs. However, it should be emphasized that our study merely provides a piece of evidence for the role of methylation status of the two miRNAs in regulating apoptosis of NSCLCs, and we should not generalize this conclusion solely based on the results from seven cell lines. To confirm this issue with sufficient data, rigorous experimental studies in more cells or in other models are definitely required in the future.

\section{Acknowledgements}

This study was supported in part by a grant from The National Natural Science Foundation of China (Grant No: 81202965). The authors declare no competing financial interests.

\section{References}

1 van Vlodrop IJ, Niessen HE, Derks S, Baldewijns MM, van Criekinge W, Herman JG, van Engeland M: Analysis of promoter CpG island hypermethylation in cancer: location, location, location! Clin Cancer Res 2011;17:4225-4231.

- Hug M, Silke J, Georgiev O, Rusconi S, Schaffner W, Matsuo K: Transcriptional repression by methylation: cooperativity between a CpG cluster in the promoter and remote CpG-rich regions. FEBS Lett 1996;379:251-254.

3 Wang Y, Leung FC: An evaluation of new criteria for CpG islands in the human genome as gene markers. Bioinformatics 2004;20:1170-1177.

Visone R, Croce CM: MiRNAs and cancer. Am J Pathol 2009;174:1131-1138.

Heller G, Fong KM, Girard L, Seidl S, End-Pfützenreuter A, Lang G, Gazdar AF, Minna JD, Zielinski CC, Zöchbauer-Müller S: Expression and methylation pattern of TSLC1 cascade genes in lung carcinomas. Oncogene 2006;25:959-968. 
6 Zöchbauer-Müller S, Fong KM, Virmani AK, Geradts J, Gazdar AF, Minna JD: Aberrant promoter methylation of multiple genes in non-small cell lung cancers. Cancer Res 2001;61:249-255.

7 Heller G, Zielinski CC, Zöchbauer-Müller S: Lung cancer: from single-gene methylation to methylome profiling. Cancer Metastasis Rev 2010;29:95-107.

8 Toyooka S, Toyooka KO, Maruyama R, Virmani AK, Girard L, Miyajima K, Harada K, Ariyoshi Y, Takahashi T, Sugio K, Brambilla E, Gilcrease M, Minna JD, Gazdar AF: DNA methylation profiles of lung tumors. Mol Cancer Ther 2001;1:61-67.

-9 Heller G, Weinzierl M, Noll C, Babinsky V, Ziegler B, Altenberger C, Minichsdorfer C, Lang G, Döme B, EndPfützenreuter A, Arns BM, Grin Y, Klepetko W, Zielinski CC, Zöchbauer-Müller S: Genome-wide miRNA expression profiling identifies miR-9-3 and miR-193a as targets for DNA methylation in non-small cell lung cancers. Clin Cancer Res 2012;18:1619-1629.

10 Watanabe K, Emoto N, Hamano E, Sunohara M, Kawakami M, Kage H, Kitano K, Nakajima J, Goto A, Fukayama M, Nagase T, Yatomi Y, Ohishi N, Takai D: Genome structure-based screening identified epigenetically silenced microRNA associated with invasiveness in non-small-cell lung cancer. Int J Cancer 2012;130:2580-2590.

11 Khodyrev DS, Pronina IV, Rykov SV, Beresneva EV, Fridman MV, Kazubskaia TP, Loginov VI, Braga ÉA:. Methylation of some miRNA genes is involved in the regulation of their target genes RAR-beta2 and NKIRAS1 expression in lung cancer. Mol Biol (Mosk) 2012;46:773-785.

12 Kitano K, Watanabe K, Emoto N, Kage H, Hamano E, Nagase T, Sano A, Murakawa T, Nakajima J, Goto A, Fukayama M, Yatomi Y, Ohishi N, Takai D: CpG island methylation of microRNAs is associated with tumor size and recurrence of non-small-cell lung cancer. Cancer Sci 2011;102:2126-2131.

13 Ceppi P, Mudduluru G, Kumarswamy R, Rapa I, Scagliotti GV, Papotti M, Allgayer H: Loss of miR-200c expression induces an aggressive, invasive, and chemoresistant phenotype in non-small cell lung cancer. Mol Cancer Res 2010;8:1207-1216.

14 Gallardo E, Navarro A, Viñolas N, Marrades RM, Diaz T, Gel B, Quera A, Bandres E, Garcia-Foncillas J, Ramirez J, Monzo M: miR-34a as a prognostic marker of relapse in surgically resected non-small-cell lung cancer. Carcinogenesis 2009;30:1903-1909.

15 Lujambio A, Calin GA, Villanueva A, Ropero S, Sánchez-Céspedes M, Blanco D, Montuenga LM, Rossi S, Nicoloso MS, Faller WJ, Gallagher WM, Eccles SA, Croce CM, Esteller M: A microRNA DNA methylation signature for human cancer metastasis. Proc Natl Acad Sci USA 2008;105:13556-13561.

16 Gao XN, Lin J, Li YH, Gao L, Wang XR, Wang W, Kang HY, Yan GT, Wang LL, Yu L: MicroRNA-193a represses c-kit expression and functions as a methylation-silenced tumor suppressor in acute myeloid leukemia. Oncogene 2011;30:3416-3428.

17 Ma K, He Y, Zhang H, Fei Q, Niu D, Wang D, Ding X, Xu H, Chen X, Zhu J: DNA methylation-regulated miR-193a-3p dictates resistance of hepatocellular carcinoma to 5 -fluorouracil via repression of SRSF2 expression. J Biol Chem 2012;287:5639-4569.

18 Kozaki K, Imoto I, Mogi S, Omura K, Inazawa J: Exploration of tumor-suppressive microRNAs silenced by DNA hypermethylation in oral cancer. Cancer Res 2008;68:2094-2105.

19 Iliopoulos D, Rotem A, Struhl K: Inhibition of miR-193a expression by Max and RXRalpha activates K-Ras and PLAU to mediate distinct aspects of cellular transformation. Cancer Res 2011;71:5144-5153.

20 Allen TD, Zhu CQ, Jones KD, Yanagawa N, Tsao MS, Bishop JM: Interaction between MYC and MCL1 in the genesis and outcome of non-small-cell lung cancer. Cancer Res 2011;71:2212-2221.

-21 Zhang H, Guttikonda S, Roberts L, Uziel T, Semizarov D, Elmore SW, Leverson JD, Lam LT: Mcl-1 is critical for survival in a subgroup of non-small-cell lung cancer cell lines. Oncogene 2011;30:1963-1968.

22 Wongpankam E, Chunhacha P, Pongrakhananon V, Sritularak B, Chanvorachote P: Artonin E mediates MCL1 down-regulation and sensitizes lung cancer cells to anoikis. Anticancer Res 2012;32:5343-5351.

-23 Starczynowski DT, Lockwood WW, Deléhouzée S, Chari R, Wegrzyn J, Fuller M, Tsao MS, Lam S, Gazdar AF, Lam WL, Karsan A: TRAF6 is an amplified oncogene bridging the RAS and NF- $\kappa$ B pathways in human lung cancer. J Clin Invest 2011;121:4095-4105.

24 Yu JA, Kalatardi S, Dohse J, Sadaria MR, Meng X, Fullerton DA, Weyant MJ: Group IIa sPLA2 inhibition attenuates NF-KB activity and promotes apoptosis of lung cancer cells. Anticancer Res 2012;32:3601-3607.

-25 Denlinger CE, Rundall BK, Jones DR: Modulation of antiapoptotic cell signaling pathways in non-small cell lung cancer: the role of NF-kappaB. Semin Thorac Cardiovasc Surg 2004;16:28-39.

-26 Cameron EE, Bachman KE, Myöhänen S, Herman JG, Baylin SB: Synergy of demethylation and histone deacetylase inhibition in the re-expression of genes silenced in cancer. Nat Genet 1999;21:103-107. 
27 Xu C, Lu Y, Lin H, Xiao J, Wang H, Luo X, Li B, Wang Z, Yang B: The muscle-specific microRNAs miR-1 and miR-133 produce opposing effects on apoptosis via targeting HSP60/HSP70 and caspase-9 in cardiomyocytes. J Cell Sci 2007;120:3045-3052.

28 Wang H, Zhang Y, Cao L, Han H, Wang J, Yang B, Nattel S, Wang Z: HERG K ${ }^{+}$channel: A regulator of tumor cell apoptosis and proliferation. Cancer Res 2002;62:4843-4848.

29 Lu Y, Xiao J, Lin H, Bai Y, Luo X, Wang Z, Yang B: Complex antisense inhibitors offer a superior approach for microRNA research and therapy. Nucleic Acids Res 2009;37:e24-e33.

30 Ma L, Young J, Prabhala H, Pan E, Mestdagh P, Muth D, Teruya-Feldstein J, Reinhardt F, Onder TT, Valastyan S, Westermann F, Speleman F, Vandesompele J, Weinberg RA: miR-9, a MYC/MYCN-activated microRNA, regulates E-cadherin and cancer metastasis. Nat Cell Biol 2010;12:247-256.

-31 Elmén J, Thonberg H, Ljungberg K, Frieden M, Westergaard M, Xu Y, Wahren B, Liang Z, Ørum H, Koch T, Wahlestedt C: Locked nucleic acid (LNA) mediated improvements in siRNA stability and functionality. Nucleic Acids Res 2005;33:439-447.

-32 Senyuk V, Zhang Y, Liu Y, Ming M, Premanand K, Zhou L, Chen P, Chen J, Rowley JD, Nucifora G, Qian Z: Critical role of miR-9 in myelopoiesis and EVI1-induced leukemogenesis. Proc Natl Acad Sci USA 2013;110:5594-5599.

-33 Tsai KW, Liao YL, Wu CW, Hu LY, Li SC, Chan WC, Ho MR, Lai CH, Kao HW, Fang WL, Huang KH, Lin WC: Aberrant hypermethylation of miR-9 genes in gastric cancer. Epigenetics 2011;6:1189-1197.

-34 Wan HY, Guo LM, Liu T, Liu M, Li X, Tang H: Regulation of the transcription factor NF-kappaB1 by microRNA-9 in human gastric adenocarcinoma. Mol Cancer 2010;9:16.

-35 Liu N, Sun Q Chen J, Li J, Zeng Y, Zhai S, Li P, Wang B, Wang X: MicroRNA-9 suppresses uveal melanoma cell migration and invasion through the NF-KB1 pathway. Oncol Rep 2012;28:961-968.

-36 Kwon JE, Kim BY, Kwak SY, Bae IH, Han YH: Ionizing radiation-inducible microRNA miR-193a-3p induces apoptosis by directly targeting Mcl-1. Apoptosis 2013;18:896-909.

-37 Lam LT, Lu X, Zhang H, Lesniewski R, Rosenberg S, Semizarov D: A microRNA screen to identify modulators of sensitivity to BCL2 inhibitor ABT-263 (navitoclax). Mol Cancer Ther 2010;9:2943-2950.

-38 Du Y, Liu Z, Gu L, Zhou J, Zhu BD, Ji J, Deng D: Characterization of human gastric carcinoma-related methylation of 9 miR CpG islands and repression of their expressions in vitro and in vivo. BMC Cancer 2012;12:249.

-39 Anwar SL, Albat C, Krech T, Hasemeier B, Schipper E, Schweitzer N, Vogel A, Kreipe H, Lehmann U: Concordant hypermethylation of intergenic microRNA genes in human hepatocellular carcinoma as new diagnostic and prognostic marker. Int J Cancer 2013;133:660-670.

-40 Shimizu T, Suzuki H, Nojima M, Kitamura H, Yamamoto E, Maruyama R, Ashida M, Hatahira T, Kai M, Masumori N, Tokino T, Imai K, Tsukamoto T, Toyota M: Methylation of a Panel of MicroRNA Genes Is a Novel Biomarker for Detection of Bladder Cancer. Eur Urol 2013;63:1091-1100.

41 Minor J, Wang X, Zhang F, Song J, Jimeno A, Wang XJ, Lu X, Gross N, Kulesz-Martin M, Wang D, Lu SL: Methylation of microRNA-9 is a specific and sensitive biomarker for oral and oropharyngeal squamous cell carcinomas. Oral Oncol 2012;48:73-78.

42 Hildebrandt MA, Gu J, Lin J, Ye Y, Tan W, Tamboli P, Wood CG, Wu X: Hsa-miR-9 methylation status is associated with cancer development and metastatic recurrence in patients with clear cell renal cell carcinoma. Oncogene 2010;29:5724-5728.

43 Nakano H, Yamada Y, Miyazawa T, Yoshida T: Gain-of-function microRNA screens identify miR-193a regulating proliferation and apoptosis in epithelial ovarian cancer cells. Int J Oncol 2013;42:1875-1882.

44 Yang Y, Li X, Yang Q Wang X, Zhou Y, Jiang T, Ma Q Wang YJ: The role of microRNA in human lung squamous cell carcinoma. Cancer Genet Cytogenet 2010;200:127-133.

-45 Caramuta S, Egyházi S, Rodolfo M, Witten D, Hansson J, Larsson C, Lui WO: MicroRNA expression profiles associated with mutational status and survival in malignant melanoma. J Invest Dermatol 2010;130:20622070.

46 Herman JG, Baylin SB: Gene silencing in cancer in association with promoter hypermethylation. N Engl J Med 2003;349:2042-5204.

-47 Lujambio A, Ropero S, Ballestar E, Fraga MF, Cerrato C, Setién F, Casado S, Suarez-Gauthier A, SanchezCespedes M, Git A, Spiteri I, Das PP, Caldas C,Miska E, Esteller M: Genetic unmasking of an epigenetically silenced microRNA in human cancer cells. Cancer Res 2007;67:1424-1429.

48 Wang Z, Chen Z, Gao Y, Li N, Li B, Tan F, Tan X, Lu N, Sun Y, Sun J, Sun N, He J: DNA hypermethylation of microRNA-34b/c has prognostic value for stage I non-small cell lung cancer. Cancer Biol Ther 2011;11:490496. 